\title{
Elementos de la conceptualización del servicio para el producto hoteles con encanto de grupo hotelero Islazul
}

\author{
Elements of the conceptualization of the service for the product charming
} hotels of the Islazul hotel group

1 Alejandro Antonio Abad Abascal

Universidad de La Habana, Facultad de Turismo, Carrera de Licenciatura en Turismo,

La Habana, Cuba

alejandro.abad@ftur.uh.cu

2 Luis Efraín Velastegui López

https://orcid.org/0000-0002-7353-5853

Universidad Técnica de Babahoyo, Babahoyo, Ecuador

evelasteguil@utb.edu.ec

Artículo de Investigación Científica y Tecnológica

Enviado: 06/12/2021

Revisado: $18 / 12 / 2021$

Aceptado: $28 / 01 / 2022$

Publicado:08/03/2022

DOI: https://doi.org/10.33262/exploradordigital.v6i1.2090

Cítese:

Abad Abascal , A. A., \& Velastegui López, L. E. (2022). Elementos de la conceptualización del servicio para el producto hoteles con encanto de grupo hotelero Islazul. Explorador Digital,

$6(1)$ 51-74. https://doi.org/10.33262/exploradordigital.v6i1.2090

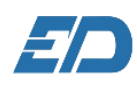

Ciencia Digital

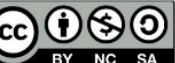

EXPLORADOR DIGITAL, es una Revista electrónica, Trimestral, que se publicará en soporte electrónico tiene como misión contribuir a la formación de profesionales competentes con visión humanística y crítica que sean capaces de exponer sus resultados investigativos y científicos en la misma medida que se promueva mediante su intervención cambios positivos en la sociedad. https://exploradordigital.org

La revista es editada por la Editorial Ciencia Digital (Editorial de prestigio registrada en la Cámara Ecuatoriana de Libro con No de Afiliación 663) www.celibro.org.ec 
Palabras claves:

beneficios, conceptualización del servicio, hoteles con encanto.

\section{Keywords:}

benefits, conceptualization of the service, charming hotels.

\begin{abstract}
Resumen
Con el propósito de alcanzar una mayor presencia en el mercado internacional y captar un mayor volumen de divisas, el Grupo Hotelero Islazul como parte de las empresas que gestionan establecimientos de alojamiento turístico en Cuba, se encuentra realizando un proceso de diversificación y perfeccionamiento de su oferta. El presente estudio pretende contribuir a dicho proceso mediante el desarrollo de la Conceptualización del Servicio para el producto hoteles con encanto perteneciente al Grupo con el propósito de perfeccionar el diseño de estas instalaciones en función de los atributos y beneficios que esperan recibir los clientes. Para ello fue realizado un análisis netnográfico de conjunto a un análisis por conglomerados jerárquicos para la determinación de los beneficios que espera la demanda de este producto hotelero y a partir de dichos beneficios de conjunto a las características de los clientes, se identificaron los atributos del servicio que deben poseer estos establecimientos. Finalmente, teniendo en cuenta las nuevas pautas en la operatoria de los hoteles impuestas por la COVID-19, fue concebida la idea del producto-servicio y los elementos de su Conceptualización.
\end{abstract}

\section{Abstract}

In order to achieve a greater presence in the international market and attract a greater volume of foreign currency, Islazul Hotel Group, as part of the companies that manage tourist accommodation establishments in Cuba, is carrying out a process of diversification and improvement of its offer. This study aims to contribute to this process by developing the Service Conceptualization for the charming hotels product belonging to the Group in order to perfect the design of these facilities based on the attributes and benefits that customers expect to receive. To this end, a netnographic analysis was carried out as a whole to an analysis by hierarchical conglomerates to determine the benefits expected by the demand for this hotel product and based on these benefits as a whole to the characteristics of the clients, the attributes of the service that they must own these establishments. Finally, taking into account the new guidelines in hotel operations imposed by COVID-19, 
the idea of the product-service and the elements of its Conceptualization were conceived.

\section{Introducción}

Según Sancho (2009), la actividad turística es resultante de complejas interrelaciones entre diferentes factores bajo una óptica sistemática, es decir, un conjunto de elementos interrelacionados entre sí que evolucionan dinámicamente, dando como resultado el sistema turístico. Su funcionamiento está relacionado a un proceso denominado ciclo de producción turística (figura 1), en el que intervienen a su vez diferentes subprocesos.

\section{Figura 1}

Ciclo de producción turística

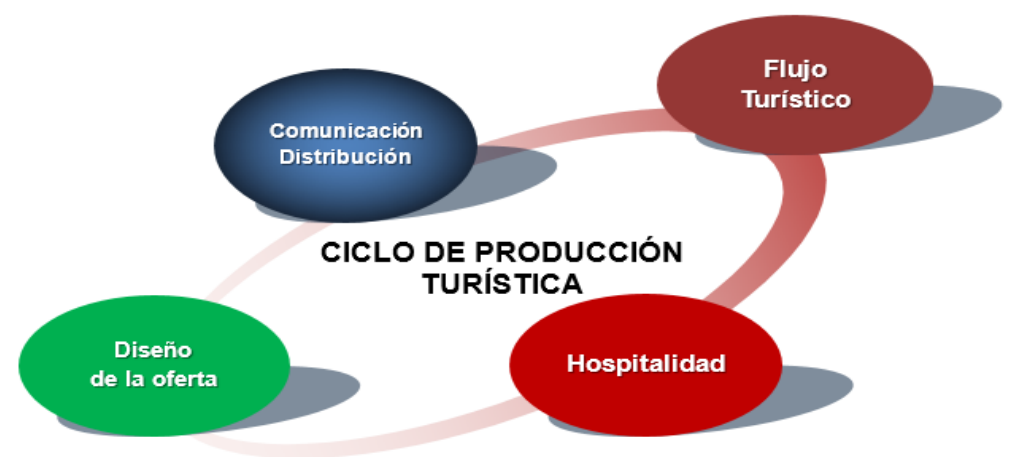

Fuente: Ulacia \& Blanco (2018)

La equilibrada interrelación de los componentes del ciclo desempeña un papel fundamental en garantizar que los clientes que consumen la oferta turística queden satisfechos y con expectativas cumplidas. Por otra parte, la adecuada concepción de los productos es otro elemento a considerar debido a que las necesidades de los consumidores son muy diversas y varían constantemente.

Autores como Valera (2019), plantean que el escenario actual se caracteriza por una fuerte competitividad y versatilidad, por lo que la apuesta por la calidad supone una ventaja competitiva y los negocios deben proyectar disímiles transformaciones en cuanto a la operación y gestión a fin de lograr la mayor coherencia posible entre la oferta y la demanda.

En cuanto a la actividad de alojamiento, existe una amplia gama de productos capaces de satisfacer las más específicas necesidades de los clientes. Como ejemplo de ello se encuentran los denominados hoteles con encanto u hoteles boutique. Estos se caracterizan 
por ser construcciones pequeñas con valor patrimonial, ubicados principalmente en los centros históricos de las ciudades. El servicio es un elemento que sobresalta por su alto grado de personalización, además de la arquitectura y decoración singular (Puig, 2015).

La actual importancia para Cuba del fenómeno turístico y sus previsiones de futuro justifican plenamente la necesidad del Grupo Hotelero Islazul de buscar nuevas oportunidades para diversificar y fortalecer su oferta, de manera que le permita incrementar su presencia en el mercado internacional, hoy cuando su principal demanda reside en el turismo interno, ya que solo el 14\% de la demanda total representa los turistas internacionales (Islazul, 2020).

La dirección de este Grupo ha solicitado el desarrollo de estudios que permitan el perfeccionamiento y la mejora de los servicios de un conjunto de 13 instalaciones que opera, denominados Hoteles E, puesto que pretende convertirlos en uno de los principales productos que posee. Estos son el resultado de adaptar viejas construcciones de carácter patrimonial, ubicadas en centros históricos de ciudades, con valor arquitectónico y cultural para ofrecer servicios de alojamiento. Se puede apreciar que dichas características están en correspondencia con lo expuesto sobre el producto denominado hoteles con encanto.

Los directivos del Grupo plantean que el desempeño de los servicios en estos establecimientos se está llevando a cabo sin conocer si se encuentran en correspondencia con los beneficios que esperan recibir los clientes que consumen el producto hoteles con encanto y tampoco existe claridad en las características de esta demanda. Por otra parte, no están precisados los atributos del servicio que deben estar presente en dichas instalaciones.

Por todo lo anterior esta investigación tiene como objetivo determinar los elementos de la conceptualización del servicio para el producto hoteles con encanto del Grupo Hotelero Islazul.

\section{Metodología}

Para el logro del objetivo propuesto fue concebida una trayectoria metodológica estructurada en tres fases.

\section{Fase 1: Fundamentación teórica que sustenta la investigación}

El desarrollo de esta fase estuvo centrado en el establecimiento de los fundamentos teóricos de la investigación en torno al tema en cuestión, determinándose los aspectos esenciales que constituyen la base del desarrollo del estudio y para ello fueron empleados los siguientes métodos y procesos del pensamiento lógico:

El método histórico se utilizó para valorar la evolución de los principales núcleos teóricos relacionados con las partes del objeto de la investigación. En el caso de este estudio, se analizó el papel de la conceptualización del servicio en el diseño de productos hoteleros, en particular los hoteles con encanto. 
De esta manera, fueron seleccionadas las teorías a tener en cuenta durante el desarrollo de la investigación. Por otra parte, el empleo del análisis-síntesis estuvo directamente vinculado a la búsqueda de las relaciones entre los diferentes aspectos conceptuales de la investigación, así como la generalización a partir de la información obtenida. También, fue empleado la inducción-deducción teniendo en cuenta el principio de garantizar una adecuada comprensión de la temática abordada de lo general a lo particular y poder transitar de lo abstracto a lo concreto.

Todo lo anterior estuvo respaldado mediante la realización de una revisión bibliográfica que tuvo en cuenta libros e informes pertenecientes a la carrera de Licenciatura en Turismo, sitios de internet que abordaban el tema en cuestión, además de revisar artículos científicos, tesis e investigaciones de autores nacionales e internacionales.

Fase 2: Determinación de los beneficios esperados por los clientes del producto hoteles con encanto

Para la determinación de los beneficios esperados por los clientes del producto hoteles con encanto fue empleado como método, que permite la recolección de datos de la demanda, el análisis netnográfico. De acuerdo con Turpo (2008, citado por Casas, 2014), este análisis posibilita un acercamiento adecuado a aquellos procesos de carácter relacional, a los comportamientos y dinámicas grupales en las comunidades virtuales en Internet.

El contexto virtual escogido para realizar el análisis fue TripAdvisor, una plataforma que asiste a 460 millones de viajeros aproximadamente cada mes, durante la planificación de sus viajes. Para determinar los comentarios de los usuarios que tomaron en el análisis, primeramente, se seleccionaron un conjunto de establecimientos hoteleros acordes a las características del producto estudiado.

Para esta selección se tuvo en cuenta las palabras claves: "hoteles con encanto" y "hoteles boutique", teniendo en cuenta que estos establecimientos, a partir del análisis teórico realizado, se conocen de esta manera. Se tomaron las 5 instalaciones de mejor posicionamiento y que cumplieran con las características siguientes:

- Construidos en edificaciones de características históricas, patrimoniales y culturales.

- Ubicados en centros urbanos.

- Pueden ser instalaciones internacionales o nacionales (la selección influye en función del posicionamiento).

Posteriormente se realizó un censo de las opiniones de los usuarios referidos a estos establecimientos en los años 2019 y 2020, dando como resultado un conjunto de 326 comentarios, los cuales fueron posteriormente analizados. Se empleó, para el procesamiento de los datos, el programa SPSS Statistics que con técnicas de agrupamiento o conglomerado 
permitió reunir casos en base a sus semejanzas a partir del cálculo realizado a los datos, donde se determinan sus distancias o índices de similaridad o disimilaridad.

Estos cálculos se realizaron aplicando el método del vecino más cercano o también conocido como algoritmo del vecino más próximo que fue, en las ciencias de la computación, uno de los primeros algoritmos destinados para determinar una solución ante el problema del viajante.

La búsqueda del vecino más próximo es una técnica muy utilizada en reconocimiento de formas y se basa en que dado un conjunto de prototipos cuya clasificación se conoce, una muestra se clasificará en la clase donde se encuentre el prototipo cuya distancia a la muestra es mínima. El criterio de segmentación para la formación de clústeres es por beneficios, ya que se consideraron como base. Por otra parte, se tuvo en cuenta para el análisis de cada grupo resultante las variables mes de visita y estilo de viaje a partir de los datos recopilados en TripAdvisor de manera que fueran descritas en la caracterización de los segmentos de demanda resultante.

Fase 3: Determinación de las pautas para el diseño de la estandarización del desempeño para los servicios de alojamiento y de alimentos y bebidas en el complejo hotelero

A partir del procesamiento y análisis de la información obtenida de las técnicas y procedimientos que fueron llevados a cabo en las fases anteriores, de conjunto a su adecuada interpretación y comprensión, se procedió a determinar los elementos que completan la conceptualización del producto-servicio estudiado.

Para la realización de lo antes expuesto, se empleó la técnica de trabajo en equipo cuyos participantes fueron las tutoras de la investigación, estudiantes de último año de la licenciatura en turismo que estuvieron involucrados en la realización del estudio y el autor del mismo. Se tuvo como base los beneficios asociados a los hoteles con encanto determinados en la Fase 2 de la investigación y también las medidas y protocolos sanitarios de estricto cumplimento, para evitar el contagio y propagación de la COVID-19.

En un primer momento se conformó el concepto del servicio para el producto estudiado y fueron puntualizados los elementos que forman parte de la idea de servicio. En un segundo momento se identificaron los atributos que requieren de especial tratamiento en este producto, dada las características de los clientes. A partir de lo anterior se determinaron, teniendo en cuenta los servicios principales del producto estudiado, los elementos tanto materiales, sensoriales y psicológicos con implicaciones relevantes para la satisfacción de los huéspedes. 


\section{Discusión}

Fundamentación teórica sobre la conceptualización del servicio y su implicación en el diseño de productos hoteleros: hoteles con encanto

Históricamente los servicios se han enmarcados como el sector terciario de la economía, pues se consideraba que no eran auténticos generadores de riquezas al constituir una actividad económica enfocada a la satisfacción de las necesidades no materiales del consumidor y no vinculada con la producción directa de bienes materiales como lo están los sectores primarios o secundarios de la economía. Por lo tanto, quedaba representado por actividades asociadas al comercio, el transporte, las comunicaciones, los servicios públicos prestados por el Estado, el turismo, entre otros.

El estudio de los mismos ha evolucionado de forma gradual y en la medida de su desarrollo han surgido diferentes enfoques. En contextos actuales, se hace necesario resaltar la definición de Ulacia (2015), que plantea que el servicio es la combinación tanto de elementos tangibles como intangibles por medio de actividades coordinadas con el propósito de producir experiencias satisfactorias en el cliente, a partir del contacto de este con el producto creado. Este enfoque concibe al servicio como un producto y, teniendo en cuenta este criterio, es de vital necesidad realizar de manera precisa su diseño.

La presente investigación parte de este enfoque del servicio como producto y del hecho de no poseer un carácter netamente intangible. Un ejemplo de ello lo constituye los establecimientos hoteleros, donde se puede apreciar claramente como los elementos materiales (tangibles) se combinan con aspectos de carácter sensoriales y psicológicos (intangibles), con el propósito de ofrecer una placentera estancia a los huéspedes, que se convierta en una experiencia diferente y recordada.

Según la Norma Cubana NC-127:2014, los hoteles son establecimientos que prestan servicio de hospedaje en unidades habitacionales amuebladas, cuentan con servicio de recepción, servicio sanitario privado, servicios de alimentos y bebidas y otros servicios adicionales.

Los requisitos y estándares de calidad de cada uno de estos servicios van en dependencia de la categoría del establecimiento, representada por un Sistema de Estrellas, establecido oficialmente por la Organización Mundial del Turismo (OMT, citado por Céspedes, 2020), autora que destaca la gran utilización a nivel internacional de dicho sistema que va desde una hasta cinco estrellas como calidad máxima.

Producto de los constantes cambios en la demanda debido a la búsqueda de experiencias novedosas y de beneficios más puntuales a la hora de satisfacer sus necesidades, la actividad turística y en especial la hotelería ha tenido que adecuarse a estas circunstancias. Los productos hoteleros han evolucionado con diseños a la medida de las características y gustos 
de clientes específicos lo que ha traído consigo un alto grado de especialización en dicho sector.

Entre los resultados de esta especialización en los servicios hoteleros podemos encontrar el producto hoteles con encanto. Según Rogerson (2010), tuvo su origen a partir de la innovación del concepto clásico de hotelería, que estuvo caracterizado por varias décadas por la estandarización sistemática. Son reconocidos por el creciente interés en el desarrollo innovador de la actividad turística y las entidades de hospitalidad que trajo consigo una especialización y altos estándares en la hotelería para las décadas de 1970 y 1980.

Estos establecimientos se caracterizan por su individualidad, por centrarse en el diseño, y se basa en operaciones de pequeño porte, en la singularidad de la estructura física, en los elevados estándares de calidad del servicio y en el cuidado individual dado a los huéspedes (Mcintosh \& Siggs, 2005).

De acuerdo con Silva \& Mota (2010), los hoteles con encanto valoran su belleza natural y su patrimonio cultural, dan importancia a los servicios de calidad y a la perfección en la atención. Además, buscan el equilibrio perfecto entre servicio, confort, comida y paisaje a través de las cinco C's: cortesía, charme (encanto), carácter, calma y cocina.

En función de lo planteado por Jones (2013), este producto hotelero es único, típicamente pequeño y con altos estándares de calidad en su servicio. Tiene un estilo propio e inspirador que lo hace ser objeto de moda y vanguardia, además ofrece una experiencia de intimidad al huésped, que combina aspectos culturales e históricos con una gama de servicios interesante.

Por otra parte, los autores Teo y Chang (2009), exponen que los clientes que consumen estos hoteles se caracterizan por ser individuos con discernimiento y distinción, debido a los elevados estándares del servicio que ofrecen. Independientemente que este producto ha alcanzado popularidad, Luján (2019) plantean que no existe del todo una homogeneidad en su definición, dando como resultado ambigüedades en sus características y elementos.

Según Ulacia \& Martínez (2017), estos son el resultado del rediseño o la creación de edificios o casas de pequeñas dimensiones, que presentan alguna característica singular para convertirlos en establecimientos de alojamiento turístico. Su principal característica es su reducida dimensión pues la gran mayoría de los mismos no cuentan con más de 30 habitaciones.

A partir de estas definiciones, puede resumirse que los hoteles con encanto ofrecen servicios de hospedaje en establecimientos que poseen un vínculo con la historia y tradiciones del lugar donde se ubican. Su arquitectura se caracteriza por la singularidad y el diseño de su oferta gira en torno a estos elementos, acompañado de un alto grado de personalización en sus servicios. Se caracterizan por la diferenciación, individualidad y detalles, además se hace referencia a estos como hoteles boutique. 
Es necesario puntualizar que estos no constituyen una tipología de establecimiento de alojamiento turístico y tampoco una modalidad de operación. Es un producto resultante de la especialización en la actividad hotelera con el propósito de satisfacer las necesidades de clientes que esperan recibir beneficios más específicos, con gustos y características particulares.

Ahora bien, lograr la satisfacción del cliente como máxima prioridad viene aparejado a mantener un equilibrio en la eficiencia operacional, a partir de un correcto diseño del producto-servicio en correspondencia con las demandas reales del mercado y el estrecho vínculo con la calidad desde su concepción, implementación y operación.

El desarrollo de la Conceptualización del Servicio posee gran importancia en el logro de lo antes planteado. En la presente investigación, dicho proceso será abordado desde el enfoque de Fisher (2011), y en la figura 2 puede observase sus fases:

Figura 2

Conceptualización del servicio

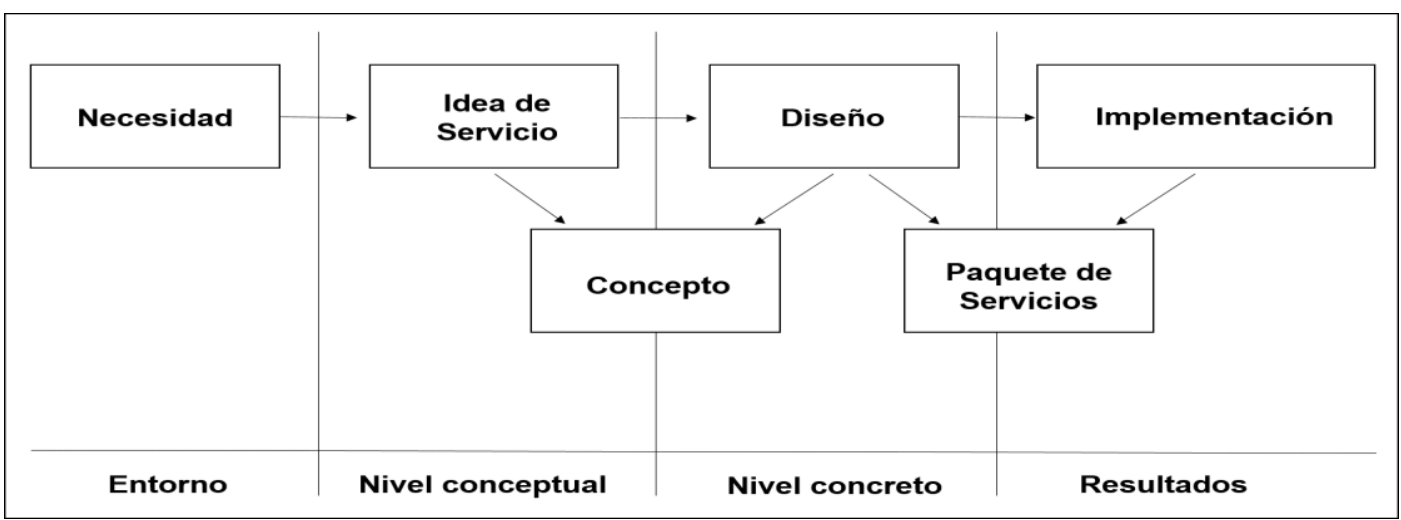

Fuente: Fisher (2011)

El despliegue de cada fase, así como la determinación de los elementos que las conforman depende de la comprensión de las expectativas de los clientes. Según Álvarez (2015), estas se encuentran directamente relacionadas a sus aspiraciones de conseguir algo específico y expone cuatro situaciones que pueden dar lugar al surgimiento de las mismas:

- Promesas que hace la misma empresa acerca de los beneficios que brinda el producto o servicio.

- Experiencias de compras anteriores.

- Opiniones de amistades, familiares, conocidos y líderes de opinión.

- Promesas que ofrecen los competidores.

Por otra parte, explica que, a partir de varios estudios, las expectativas tienen un basamento en aspectos tales como: adquirir un producto o servicio que satisfaga su necesidad o su deseo, 
obtener su satisfacción al menor precio posible, mínimo de esfuerzos, complicaciones y averías posible y en recibir un trato agradable por parte del serviciador.

A partir de estos elementos es necesario comprender que el cumplimiento de las expectativas de los clientes juega un papel fundamental en su satisfacción, por lo que resulta imprescindible estudiarlas a fondo. Dada las características propias de un servicio, la relación entre percepciones y expectativas es relativa a cada cliente en particular, son estos quienes las determinan, no el prestador del servicio. Sin embargo, los negocios pueden influir en la satisfacción del cliente intentando cumplir con sus expectativas (superándolas en el mejor de los casos).

Junto al hecho de que el cliente está buscando satisfacer esa necesidad, se manifiestan procesos de carácter afectivo que intervienen en su búsqueda, por lo que no sólo se trata de tener ese algo, sino también de saber cómo se ofrece para satisfacer los elementos de carácter material junto con las sensaciones y experiencias, desde el punto de vista emocional, que está deseando vivir al entrar en contacto con el servicio.

En concordancia con lo analizado anteriormente, es posible afirmar que las necesidades son el punto de partida para conceptualizar y diseñar un servicio. A partir de su identificación, la empresa debe enfocarse en concebir productos capaces de cumplir con las expectativas de los clientes.

\section{Fase de análisis de necesidades}

Es de vital importancia partir de estudios de demanda, con el propósito de identificar posibles segmentos de clientes que posean necesidades insatisfechas, o por lo menos, no cubiertas del todo. Estas brechas en el mercado permiten a los prestatarios del servicio concebir productos a partir de la determinación de aquellos beneficios que esperan dichos clientes.

Los beneficios según Gotteland \& Hoan (2005), son el conjunto de las ventajas o satisfacciones que un producto proporciona a las necesidades o deseos de los consumidores. La diferenciación entre los segmentos está dada por diversas características condicionadas por factores como la edad, forma de vida, lugar donde viven, entre otras, que hacen difícil diseñar productos-servicios capaces de satisfacerlos a todos.

Levy (1998), considera que la investigación de la demanda es la búsqueda de información sobre el consumidor final, es el conocimiento de los atributos que espera, del origen de los demandantes, del tipo de bienes y servicios que solicitan, de las tendencias especiales de desplazamiento.

Para caracterizar la demanda, debe tenerse en cuenta en principio el propio concepto de demanda. Esta surge, desde el punto de vista individual, a partir de que las personas tengan 
como mínimo la necesidad o interés de viajar, el tiempo disponible para realizar la actividad y, el nivel de renta adecuado o financiamiento para los gastos del viaje (Martín, 2009).

La segmentación según Kotler \& Armstrong (2013), se basa en la diversidad de los consumidores y afirman que, por consiguiente, existirán diferentes demandas por cada grupo relativamente homogéneo de consumidores. En pos de garantizar que la segmentación realmente sea útil, estos autores plantean que los segmentos deben ser:

- Medibles: Pueden medirse el tamaño, el poder adquisitivo y los perfiles de los segmentos.

- Accesibles: Los segmentos de demanda pueden ser efectivamente alcanzados y atendidos.

- Sustanciales: Son lo suficientemente grandes o rentables para atenderlos. Un segmento debe ser el grupo homogéneo más grande posible en el que valga la pena dirigir un programa de marketing a la medida.

- Diferenciables: Los segmentos son conceptualmente distinguibles y responden de manera diferente a los diversos programas y elementos de la mezcla de marketing.

- Abarcables: Es posible diseñar programas eficaces para atraer y atender a los segmentos.

La segmentación es muy útil para identificar posibles negocios, permitiendo ajustar la oferta de sus productos a las exigencias de la demanda. Además, contribuye a establecer prioridades y la toma de decisiones de carácter económico y comercial (Serra, 2003).

\section{Fase Conceptual}

La fase conceptual (nivel conceptual) es donde se genera la idea del servicio a poner en el mercado y se determina el Concepto del mismo como elemento principal para el desarrollo del producto-servicio. Se concibe cada aspecto que formará parte del producto y las formas en las que serán dispuestos, de manera que se pueda visualizar como será y cuáles son sus características.

El Concepto del producto-servicio juega un papel fundamental para su posterior desarrollo, pues actúa como guía para integrar de manera armónica cada uno de los elementos empresariales. Este es definido por Ulacia (2015), como las intenciones de la organización en términos de beneficios a ofrecer al segmento de clientes para el cual se diseña el sistema de servicios, en pos de construir una posición que sea sólida y, a su vez, potencialmente flexible, que posibilite el logro de los objetivos, a pesar de los cambios del entorno.

Fase de materialización o nivel concreto:

La idea de servicio comienza a materializarse a través del paquete de servicios. Esta herramienta muestra de manera concreta todos los elementos materiales, sensoriales y 
psicológicos, que serán puestos a disposición de los clientes. Se hará especifico las características de estos, de manera que sean elementos que aporten ventajas competitivas y no posean un carácter asumido.

La existencia de servicios complementarios o de apoyo, también conocidos como periféricos o secundarios que complementan y enriquecen el producto principal, condiciona que se determinen mayor número de elementos tangibles e intangibles. Este hecho conlleva a que se confeccionen paquetes, en función de la cantidad de servicios que ofrece la instalación, siempre enmarcando la diferenciación de aquellos servicios que posean un carácter principal.

\section{Fase de resultados}

Esta fase es el momento de la implementación o entrega del servicio, donde el cliente evalúa la satisfacción de sus expectativas. Según Cartaya (2015), la satisfacción del cliente es el resultado de la comparación que de forma inevitable se realiza entre sus expectativas previas puestas en los productos-servicios que se promocionan y en los procesos e imagen de la empresa, con respecto al valor percibido al finalizar la relación comercial.

Referido a este tema Álvarez (2015), plantea que la satisfacción implica:

- La existencia de un objetivo que el cliente desea alcanzar.

- La consecución de este objetivo, sólo puede ser juzgada tomando como referencia un estándar de comparación.

- El proceso de evaluación de la satisfacción implica como mínimo la intervención de dos estímulos: un resultado y una referencia o estándar de comparación.

La determinación de la idea del servicio, a partir del concepto y el desarrollo de la oferta mediante los componentes del paquete de 'servicio, deben estar acordes al cumplimiento de atributos basados en el estudio de las necesidades de los clientes. Fisher (2011), plantea que los atributos del servicio, a los cuales también llama como adjetivos polarizantes del servicio, son los siguientes:

- Seguridad: Una de las exigencias más fuertes de los clientes de hoy, se refriere a la seguridad tanto de su integridad física como de sus propiedades. Dentro de este atributo, entran también los aspectos relacionados con el cuidado del medioambiente, de ahí que muchas empresas, en particular, las turísticas, muestren una gran preocupación por integrar a su gestión los elementos que guardan relación con este aspecto, desde la elaboración de Manuales hasta la opción de premios, pasando por las certificaciones en este tema.

- Consistencia: La consistencia, como atributo del servicio, significa la estandarización del mismo. Es decir, la manera en que se presta el servicio, o cómo se ofrece el producto, es siempre la misma, sin cambios significativos. Cuando un servicio es consistente, el cliente sentirá confianza en cada uno de los momentos en 
que decida ir a buscarlo. Cuando esto no sucede, la credibilidad de la empresa en términos de satisfacción, se resiente y el cliente crea un sentimiento de inseguridad difícil de borrar.

- Actitud: Se centra en la relación interpersonal fundamentalmente, entre el personal de contacto y el cliente. Es quizá el atributo al que más se hace referencia, en tanto elementos como la amabilidad, la empatía, la cortesía, presencia personal, etc. son los que reciben una mayor atención de las empresas.

- Integralidad: Significa el grado de diversidad de servicios afines ofrecidos en el sistema global de prestación. La integralidad guarda una estrecha relación con el concepto de servicios complementarios o periféricos por lo que es uno de los atributos más necesitados de tenerse en cuenta, en el momento del diseño.

- Ámbito: Se refiere a la atmósfera, el ambiente en el que se ofrece y produce el servicio. Este atributo se asocia a los beneficios sensoriales que conforman el paquete de servicios y la creación de experiencias para el cliente.

- Disponibilidad: La accesibilidad, en tiempo y espacio, de un servicio, se agradece grandemente por los clientes. La misma, se traduce en la proximidad del servicio a los clientes, lo que no sólo va a referirse a la ubicación o localización del servicio, sino también, a horarios adecuados, etc.

- Oportunidad: Todo servicio debe prestarse a tiempo. Este atributo no se refiere precisamente a la rapidez, sino al momento en que se precisa.

- Adaptabilidad: Se refiere a la flexibilidad que debe presentar el servicio para que sea adecuado al cliente tanto en la oferta como en el proceso.

- Costo: Los estudios en relación a lo que más valoran los clientes en la prestación de un servicio, han señalado en último lugar el costo. En la perspectiva del cliente, el costo, se refiere tanto al precio, como al tiempo y el esfuerzo que hacen para recibir el servicio. Por ejemplo, realizar un viaje de 10 horas desde Europa hasta Cuba, con el correspondiente cansancio que esto produce y el precio pagado para el mismo, será para el cliente un elemento de poco impacto en su experiencia, si durante su estancia, el resto de los atributos antes mencionados, están presentes en el producto-servicio que han comprado.

La materialización de los beneficios y el cumplimiento de los atributos, ya sean los genéricos u otros atributos más específicos que resulten del estudio, impactan directamente en el nivel de servicio de la organización. Desde la perspectiva del cliente o el comprador, es identificado como la evaluación de estos sobre la calidad del servicio, a partir de su percepción y plantea que puede adquirir tres formas: el nivel de servicio ofrecido, el nivel de servicio entregado y el nivel de servicio percibido por el cliente.

El objetivo de la entidad es hacerlos coincidir principalmente el servicio entregado y el percibido y para ello es de vital necesidad prestarle atención a la determinación de estándares que permitan el cumplimiento de las características relevantes que se originan de la 
combinación de atributos percibidos por el cliente. Este, a partir de identificarlos en el producto-servicio, los evaluará en cada uno de los momentos en que tenga contacto con la empresa.

Determinación de los beneficios esperados por los clientes del producto hoteles con encanto

Del análisis de los hoteles seleccionados para la identificación de los beneficios esperados respecto a este tipo de producto, pudo apreciarse que prácticamente en la mayoría de las instalaciones analizadas, está presente el servicio de recepción 24 horas. Por otra parte, se observa la presencia del servicio de conserjería, también el acceso a internet gratuito y las habitaciones se encuentran insonorizadas.

Como parte del análisis netnográfico se identificaron elementos genéricos de cada comentario que fueron perfilados y seleccionados aquellos que correspondían a beneficios y estaban presente en más del $50 \%$ de las opiniones.

Quedaron definidos un conjunto de ocho beneficios mencionados a continuación:

- Beneficio 1: Elegancia

- Beneficio 2: Decoración autóctona de la cultura

- Beneficio 3: Ambiente de calma y tranquilidad

- Beneficio 4: Ambiente de intimidad

- Beneficio 5: Atención personalizada

- Beneficio 6: Conocimientos de la cultura y tradiciones del lugar

- Beneficio 7: Oferta gastronómica típica del lugar

- Beneficio 8: Servicio gastronómico personalizado

Como se puede apreciar en la Figura 3, de los ocho beneficios identificados el más esperado y presente en el $100 \%$ de los comentarios es la oferta gastronómica típica de lugar, seguido por la atención personalizada en un $98 \%$ de las opiniones. Por otra parte, como resultado del análisis por conglomerados, se obtuvieron dos clústeres (dos segmentos de la demanda) que resultan de interés para el desarrollo de esta investigación. 


\section{Figura 3}

Beneficios identificados en el análisis netnográfico

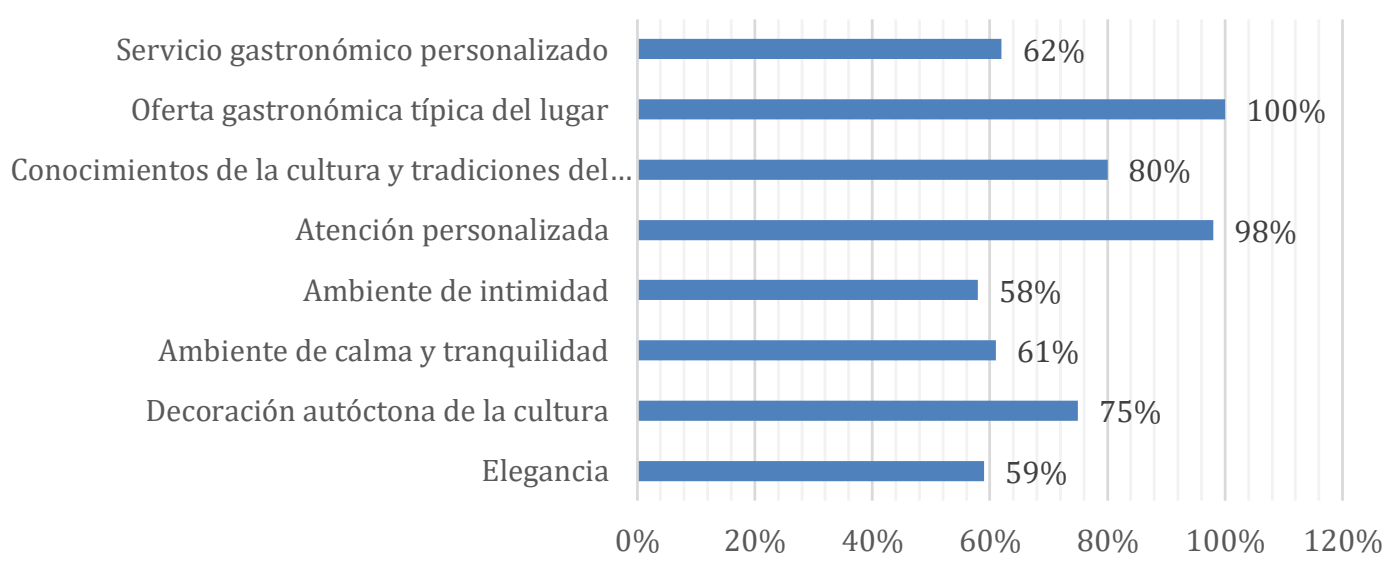

La figura 4 realiza una comparación del comportamiento de los beneficios entre los dos segmentos identificados (dos clústeres resultantes) en la demanda del producto hoteles con encanto, resaltando que el segmento 1 está representado por el color naranja y el Segmento 2 por el azul. Los resultados de esta comparación se encuentran reflejados en las características de cada segmento.

\section{Figura 4}

Comportamiento de los beneficios por segmentos

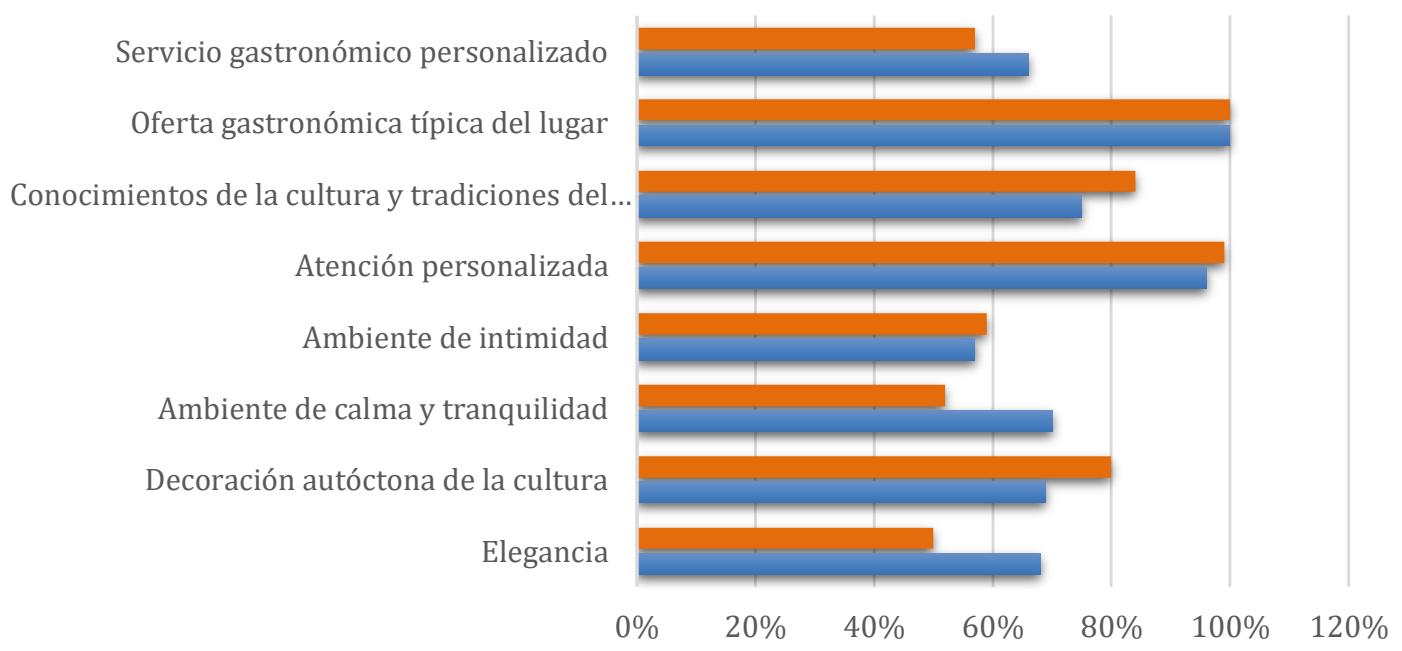

Segmento 1 (Naranja):

Este segmento representa el $52 \%$ de los casos analizados y se caracterizan por viajar mayormente en dos trimestres del año, diciembre-febrero (45\%) y septiembre-noviembre (40\%) y, en menor medida, junio-agosto con un 15\%. Predomina el estilo de viaje Pareja 
(45\%) que, aunque no supera la mitad del segmento, es más representativo que los demás estilos.

Características:

- Le conceden gran importancia a que el personal de servicio ofrezca un trato personalizado.

- Se muestran interesados por conocer la cultura y tradiciones del lugar donde se encuentra el establecimiento y también valoran que toda la decoración y ambientación de la instalación sea en función de esto.

- Prefieren una oferta gastronómica acorde con los elementos autóctonos de la localidad.

- Tienen una tendencia al gusto por ambientes de intimidad.

Segmento 2 (Azul)

Este representa el $47 \%$ de los casos analizados y se caracterizan por viajar mayormente en el trimestre de marzo-mayo (58\%), aunque existe un porciento que viaja en el de junioagosto $(41 \%)$. Posee un marcado predominio del estilo de viaje Pareja (65\%) sobre el resto de los estilos.

Características:

- Aprecian la atención personalizada por parte del personal de servicio.

- Valoran la elegancia en toda la decoración y ambientación del establecimiento y los espacios de calma y tranquilidad.

- Buscan disfrutar de una oferta de alimentos y bebidas caracterizada por las costumbres y tradiciones típicas del lugar donde se encuentra la instalación y que exista un servicio gastronómico con carácter personalizado.

A partir de los beneficios identificados y las características de los segmentos resultantes se determinaron, para el producto analizado, los atributos del servicio que se muestran a continuación:

- Ámbito

Los elementos que conforman la decoración de todas las áreas del establecimiento deben ser cuidadosamente seleccionados acordes con las tradiciones culturales de la localidad y su historia, resaltando los valores patrimoniales que tienen los establecimientos, predominando la elegancia. Por otra parte, la música, la iluminación y los colores deben estar en función de estos valores histórico-culturales.

- Actitud 
El personal de servicio tiene la tarea de, además de ofrecer un trato excelente a los huéspedes, transmitir conocimientos referidos a los valores histórico-culturales de la instalación y el lugar donde se encuentra y también de las tradiciones que existen en la localidad. Por otra parte, juega un papel fundamental en ofrecer un servicio gastronómico caracterizado por un trato diferenciado y exclusivo que trasmita una sensación de importancia a los clientes.

- Adaptabilidad

La concepción que debe tener el personal de contacto respecto a que el servicio debe ser flexible y que es necesario buscar alternativas en función de las preferencias del cliente, influye en gran medida en ofrecer un servicio con alto grado de personalización.

- Consistencia

Para el mantenimiento de una oferta gastronómica compuesta por elaboraciones típicas del lugar donde se encuentra el establecimiento, se hace necesario prestarle una estrecha atención a la selección de los proveedores.

- Integralidad

La ambientación y decoración en torno de la cultura local en concordancia con la oferta de servicios gastronómicos.

Elementos de la conceptualización del servicio para el producto hoteles con encanto del grupo Hotelero Islazul

Durante el desarrollo de esta investigación se ha podido determinar que, entre las características fundamentales de este tipo de establecimientos, resaltan el hecho de que son edificaciones con valor patrimonial y son instalaciones de pequeño formato, lo cual resulta beneficioso para desarrollar un ambiente acogedor y con alto grado de personalización en el servicio.

\section{Idea del Producto-Servicio}

Se hace necesario, para el adecuado diseño del producto-servicio, puntualizar en los siguientes elementos:

- Personalización (Servicio adaptado y adecuado a los requerimientos del cliente, conocimiento de los gustos y deseos de los mismos a partir del momento de realización de la reserva en pos de garantizar la experiencia más satisfactoria, haciendo énfasis en los detalles que puedan causar excelentes momentos de contacto)

- Ambientación (Decoración con elementos autóctonos más específicos del lugar donde se encuentran las instalaciones y teniendo en cuenta su historia, en armonía 
con elegancia, intimidad y un ambiente de calma que garantice el bienestar de los huéspedes a través de adecuadas condiciones físicas de los inmuebles)

- Historia, tradición y cultura (Conocimiento de la cultura, historia y tradiciones de la ciudad y la localidad donde se encuentran las instalaciones, que el cliente no sienta que el hotel solo es el lugar donde dormirá, sino que sea parte fundamental en la experiencia que desea tener en el lugar y punto de referencia para la realización de sus actividades)

Teniendo en cuenta estos aspectos, cada área de los establecimientos debe ser nombrada con elementos autóctonos de la cultura de la localidad, guardando un vínculo estrecho con la historia de cada instalación. Los equipos o accesorios de la modernidad deben estar situados de manera armónica, de forma tal que pasen desapercibidos y el ambiente esté bien equilibrado.

Es importante la presencia de espacios para realizar actividades como lectura, relajarse escuchando música, entre otras, donde puedan ser ofrecidos servicios de bebidas, coctelería y alimentos ligeros. Debe existir un área de venta, bien ubicada en concordancia con el entorno del establecimiento, donde se ofrezcan productos típicos de Cuba (tabaco, café cubano, artesanías y ron).

De acuerdo con la norma cubana NC-127:2014, que regula los requisitos para la clasificación por categorías de los establecimientos de alojamiento turístico y por consiguiente la actividad de los mismos en Cuba, si las instalaciones no son 5 estrellas, no tienen como requisito obligatorio la inclusión de la figura del conserje. Sin embargo, las funciones asociadas a la conserjería deben ser asumidas por todo el personal de servicio con el propósito de ofrecer un trato con alto grado de personalización a los clientes. De esta manera, cada trabajador debe tener la concepción de satisfacer a los huéspedes independientemente de la función o el puesto que desempeña.

Respecto a los servicios gastronómicos, el vínculo que se debe crear entre el personal de servicio y los clientes es muy importante en este tipo de productos. Los huéspedes buscan alto grado de personalización, pero este será en concordancia a la nueva realidad impuesta por la COVID-19. El principal reto es ofrecer ese servicio cercano de manera que no sea un medio para la transmisión y el contagio del SARS-CoV-2.

El desayuno es el mayor contacto que tienen los clientes con los servicios asociados al área de alimentos y bebidas en función de la oferta que se vende en estos establecimientos (Hospedaje + Desayuno). Teniendo en cuenta que los clientes esperan recibir un servicio en contacto con la localidad y, dentro de los protocolos sanitarios para enfrentar la COVID-19, se recomienda no estar en lugares cerrados, es importante acondicionar los patios y terrazas de los establecimientos para el desempeño del servicio de desayuno en los horarios establecidos para su realización. 
La existencia de soluciones desinfectantes y kits de aseo para los clientes es imprescindible, además de los correspondientes pasos podálicos a la entrada y salida de los locales del servicio.

La entrega oportuna del servicio requiere que todo el personal tenga presente el cumplimiento de los elementos siguientes:

- Asegurar la higiene adecuada en el entorno para el servicio, en el soporte físico, en los utensilios y otros elementos físicos necesarios para la producción del servicio/experiencia, en los productos, y en la presentación del personal.

- Cumplir con los conceptos de cortesía, respeto y trato amistoso imprescindible en la atención al cliente, mostrando un verdadero y comprometido interés por ayudarle y servirle.

- Garantizar en todo momento la seguridad del cliente y de sus pertenencias.

- Nunca ofrecer un no como respuesta ante una solicitud de los clientes, buscando en todo momento la alternativa a su requerimiento.

- Conocimiento de la cultura local y del país, las principales manifestaciones artísticas de nuestro pueblo.

- Conocimiento de cada servicio que se ofrece en el hotel.

- El personal de servicio debe comportarse de manera adecuada en las áreas de la instalación, manteniendo un tono de voz bajo, con expresiones corporales correctas ante los clientes.

- Debe identificar por el nombre a cada cliente que se encuentre hospedado con el objetivo de ofrecer un trato personalizado, estableciendo para ello un sistema que permita garantizarlo.

- El personal de apoyo no debe estar fuera de su área, solo en las ocasiones que sea necesario transitará por las áreas donde se encuentran los clientes manteniendo una correcta forma de actuar y un tono de voz adecuado.

\section{Concepto del Producto-Servicio}

Estancia con alto grado de personalización, elegancia y autoctonía espirituana en un ambiente que propicia intimidad y tranquilidad.

Para el servicio asociado a la actividad de alojamiento es necesario resaltar los siguientes pertenecientes a los componentes tangible e intangible:

Materiales

- Mesa de madera de estilo colonial (mostrador de la Recepción).

- Muebles de estilo colonial asociados a la época de construcción de cada instalación.

- Cuadros con temática colonial de autores de la región. 
- Esculturas de diferentes tamaños en concordancia con la historia de la ciudad en la etapa colonial.

- Plantas ornamentales representativas de la región.

- Búcaros, ceniceros, floreros con diseños acordes a la época de las instalaciones y la historia de la ciudad.

- Lámparas de estilo colonial en equilibrio con la decoración.

- Dispensadores de soluciones desinfectantes a base de alcohol con diseños armónicos con la temática decorativa de cada instalación.

Sensoriales

- Empleo de la iluminación natural combinada con la artificial (color amarillo que predominaba en la luminaria de la época colonial).

- Reproducción de música cubana tradicional con un volumen adecuado, haciendo énfasis en artistas locales.

- La disposición de cada elemento debe garantizar un distanciamiento de $1,5 \mathrm{~m}$ entre las personas.

- El personal de las instalaciones debe tener la concepción de que, independientemente de su plaza o función, se encuentra a disposición de los huéspedes para cualquier solicitud que estos realicen. El uniforme tiene que estar en concordancia a los colores que están presentes en las instalaciones, por lo tanto, puede ser una combinación entre amarillo y carmelita y, además, debe poseer el logo de los hoteles.

Psicológicos

- Sensación de estar en una época diferente a la era moderna (Época Colonial Cubana).

- Sensación de importancia dada por el alto grado de personalización del servicio y la elegancia y clase del ambiente de las instalaciones.

Para el servicio asociado a la oferta de desayuno es necesario resaltar los siguientes pertenecientes a los componentes tangible e intangible:

Materiales

- Mesas y sillas de estilo colonial con capacidad máxima para cuatro personas.

- Cuadros y elementos decorativos que sean alegóricos a la época e historia de las instalaciones.

- Lámparas alegóricas a la época colonial y acordes a la historia de las instalaciones.

- Vajilla decorada con el logo de los Hoteles E.

- Oferta compuesta por:

- Café de marcas cubanas y como mínimo tres variantes de preparación (americano, expreso y cappuccino).

- Leche. 
- Jugo de frutas naturales (tres tipos de jugos según temporadas a base de frutas representativas Cuba, específicamente de la región de las instalaciones).

- Dos tipos yogurt (natural y con sabor de las frutas más representativas de Cuba).

- Pan de molde (puede ser presentado en tostadas) y baguette, acompañado de mantequilla, jamón, queso.

- Frutas y vegetales (tres tipos de frutas y dos de vegetales según estación predominando los productos representativos de la región de las instalaciones).

- Huevos (cuatro tipos de preparación: tortilla, revueltos, fritos o cocidos).

- Postres representativos de la gastronomía local y cubana en sentido general.

- Recipientes con atomizador que contengan soluciones desinfectantes a base de alcohol.

- Pasos podálicos a la medida de las entradas y salidas de los locales donde se realiza el servicio.

Sensoriales

- La disposición de las mesas y sillas debe garantizar un distanciamiento de $1,5 \mathrm{~m}$ entre las personas.

- Uso de luz natural en combinación con iluminación artificial (color amarillo que predominaba en la luminaria de la época colonial).

- Reproducción de música cubana perteneciente a géneros de diferentes épocas con un volumen adecuado, haciendo énfasis en artistas locales.

- Acompañamiento musical por trío de guitarras.

- Música solo cubana y representativa de las más autóctonas tradiciones culturales de la ciudad de los establecimientos y del país en sentido general.

- Los dependientes de servicio gastronómico deben reconocer a los huéspedes, de manera que, con adecuado respeto, establezcan un vínculo que permita una prestación con alto grado de personalización.

Psicológicos

- Sensación de extrapolación a la época de la colonia cubana acompañada de una sensación de importancia producida por la elegancia de la ambientación y por el servicio personalizado.

\section{Conclusiones}

- La fundamentación teórica realizada posibilitó comprender que el desarrollo de las cuatro fases de la Conceptualización del Servicio permite la concepción y diseño de 
productos hoteleros, en este caso los hoteles con encanto, a partir de un enfoque centrado en el Servicio.

- Quedaron identificados como resultado del estudio de la demanda de los hoteles con encanto un conjunto de ocho beneficios entre los que sobresalen la atención personalizada, la oferta gastronómica típica del lugar y decoración autóctona de la cultura, destacándose entre las características de los clientes el estilo de viaje Pareja.

- A partir del Concepto de Servicio propuesto se diseñaron los principales elementos materiales, sensoriales y psicológicos en los servicios asociados al alojamiento y a la oferta de desayuno, en pos de garantizar la entrega de los ocho beneficios determinados en el estudio.

\section{Referencias bibliográficas}

Álvarez, D. (2015). Medición de la satisfacción del cliente en el restaurante Rancho Palco. Trabajo de Diploma en Opción al Título de Licenciado en Turismo. La Habana.

Cartaya, D. (2015). Causas que inciden en el bajo nivel de ingreso por concepto de comestibles en la cafetería Piropo Jesús María de la Sucursal Palmares Centro. Trabajo de Diploma en Opción al Título de Licenciado en Turismo. La Habana.

Casas, A. (2014). La netnografía como herramienta de investigación en contextos online: una aplicación al análisis de la imagen de los servicios públicos de transporte. Innovar Vol. 24. Bogotá.

Céspedes, A. (2020). Diagnóstico a la satisfacción de clientes externos en el Hotel Memories Miramar. Trabajo de Diploma en Opción al Título de Licenciado en Turismo. La Habana.

Fisher Angulo, R. (2011). El Desafío del Servicio. Versión 2.0 (corregida y ampliada). Editorial HSU (Hospitality \& Service University). Santiago de Chile.

Gotteland \& Hoan (2005). Developer un nouveau product: methods et outlies. Pearson Education France. France.

Islazul, G.H. (2020). Balance de trabajo anual 2019. La Habana.

Jones, D.L. (2013). Emerging Definitions of Boutique and Lifestyle Hotels: A Delphi Study. Journal of Travel \& Tourism Marketing.

Kotler \& Armstrong (2003). Fundamentos del Marketing. México: Pearson Educación.

Levy, A. (1998). Mayonesa, la Esencia del Marketing: Principios Fundamentales del Desarrollo Competitivo. Buenos Aires: Granica, S.A. 
Luján, M. (2019). Factores determinantes de la competitividad en hoteles boutique. El Periplo Sustentable Universidad Autónoma del Estado de México ISSN: 1870-9036, Número: 36

Martín, R. (2009). Principios Organización y Prácticas del Turismo. La Habana: Félix Varela.

Mcintosh, A. \& Siggs, A. (2005). An exploration of the experiential nature of boutique accommodation. Journal of Travel Research 44(1): 74-81

Puig, A. (2015). Las Nuevas Modalidades de Alojamiento: hoteles boutique, hoteles con encanto, hoteles Feng-Shui. Madrid.

Rogerson, J. (2010). The boutique hotel industry in South Africa: definition, scope, and organization. Urban Forum 21(4): 425-439

Sancho, A. (2009). Introducción al Turismo. Organización Mundial del Turismo.

Serra. (2003). Marketing Turístico. Madrid: Pirámide.

Silva, D. R. F. \& Mota, K. C. N. (2010). O segmento da hotelaria de charme como estratégia mercadológica no ceará. I Encuentro Semintur Jr, Universidad de Caxias do Sul, Caxias do Sul

Teo, P. \& Chang, T. C. (2009). Singapore's postcolonial landscape: boutique hotels as agents. In: Winter, T.; Teo, P. \& Chang, T. C. (Eds.) Asia on tour: exploring the rise of Asian tourism. Routledge, Abingdon, pp. 81-96

Ulacia, Z. (2015). Sistemas de Servicio y Hospitalidad. La Habana: Félix Varela.

Ulacia, Z y Martínez, Y. (2017). La Gestión Comercial del Hotel. Gestión de Alojamientos Capítulo 3. La Habana.

Ulacia, Z. y Blanco, G. (2018). Establecimientos de alojamiento turístico. Quito: Humus.

Valera, A. (2019). Diseño de la oferta de desayuno del Hotel Paseo Habana para una modalidad de operación Bed\&Breakfast. Trabajo de Diploma en Opción al Título de Licenciado en Turismo. La Habana.

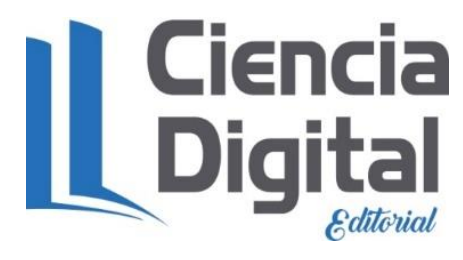




\section{EPDigital}

El artículo que se publica es de exclusiva responsabilidad de los autores y no necesariamente reflejan el pensamiento de la Revista Explorador Digital.

\section{¿Ciencia}

El artículo queda en propiedad de la revista y, por tanto, su publicación parcial y/o total en otro medio tiene que ser autorizado por el director de la Revista Explorador Digital.
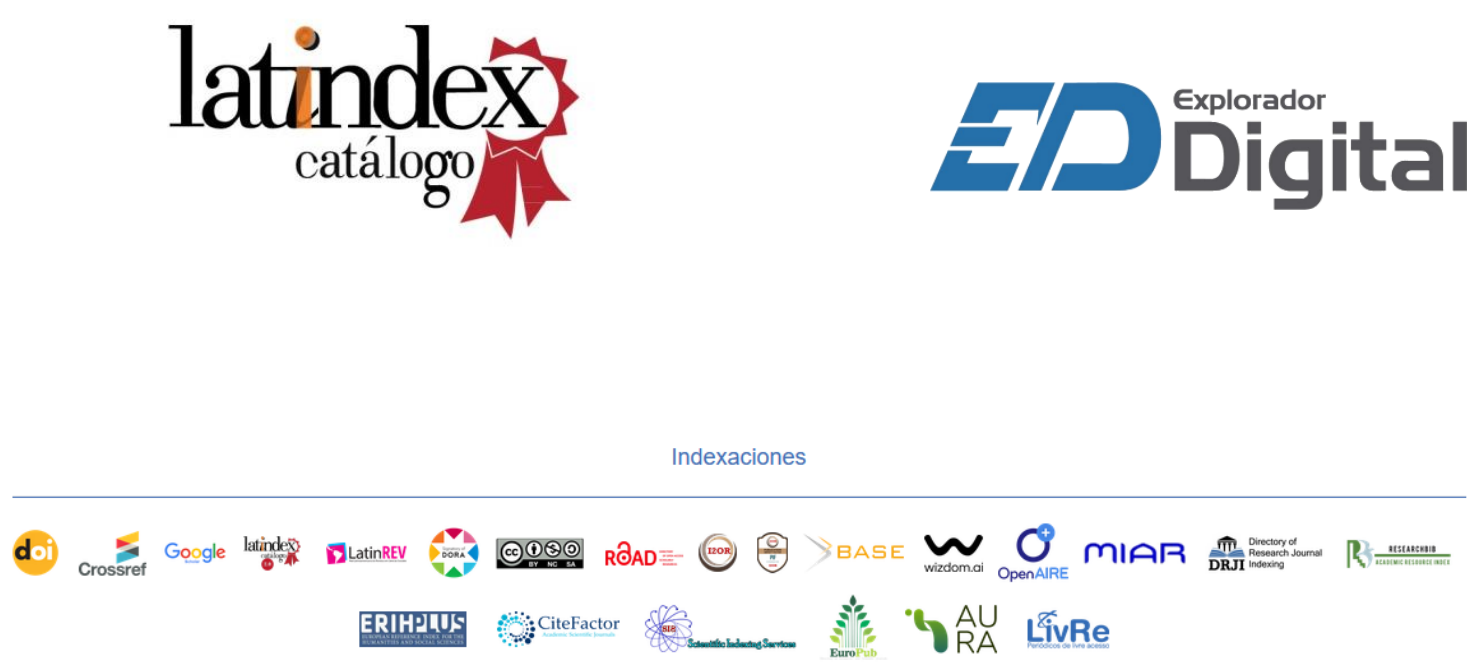